\title{
PolimiparaRocinha: Environmental Performances and Social Inclusion-A Project for the Favela Rocinha
}

\author{
Gabriele Masera, Massimo Tadi, Carlo Biraghi and Hadi Mohammad Zadeh
}

\begin{abstract}
By the middle of the current century, the world's population is projected to grow exponentially, becoming one of the major concerns for the built environment all around the world, where informal settlements are going to grow even faster. This growth will increase the demand for basic infrastructure which is lacking in such contexts. In developing countries, the promotion of urban technologies could contribute immensely to a sustainable development and to population well-being, besides creating interesting economic opportunities. Urban technologies could reduce the environmental impact of cities' development and of urbanized slums renovation, creating employment opportunities for locals and economic opportunities for investment. However, deploying this in slums is a complex and challenging task. This text presents a project based on a multidisciplinary and integrated design methodology for the sustainable regeneration of Rocinha, one of the largest favelas of Rio de Janeiro. The project adopts a systemic approach and foresees the deployment of an urban management system (UMS) able to manage and integrate several urban services including sanitation, energy, mobility, waste, food delivery and cultivation, and the flow of information connected to them, with the aim of reducing the environmental impact while improving the quality of life of citizens. Each of these areas required the development of a specific project that, empowered by the UMS, will allow for the circulation of information between citizens, fostering social inclusion, and raising awareness on the topic of the city's resource management. This project is a demonstration of how minimal but calculated local modification can produce considerable global reaction and ultimately change the system as a whole.
\end{abstract}

Keywords Favela $\cdot$ Informal settlements $\cdot$ System thinking $\cdot$ Complex adaptive system $\cdot$ Urban management system $\cdot$ Multiscale approach

G. Masera ( $\varangle) \cdot$ M. Tadi · C. Biraghi · H. M. Zadeh

Architecture, Built Environment and Construction Engineering-ABC Department, Politecnico di Milano, Milan, Italy

e-mail: gabriele.masera@polimi.it 


\section{Introduction}

As the urban population of the world is increasing dramatically, it is predicted that by the year 2050 about $70 \%$ of this population will live in informal settlements. While this is an alarming matter for policy makers and urban managers around the world to revise and replan development, it highlights the importance of studying such organizations for scientific communities too.

Depending on the local environment, cultural context, size, and the contextual relationships with the surrounding urban areas, the informal settlements could appear in a variety of shapes and forms. Nonetheless, there are structural features shared among them worldwide. There are innate characteristics such as high population densities, poor infrastructures, and social segregation associated with them which make them much more challenging to manage, and at the same time, there are also surprising peculiarities, like certain environmental behaviors, offered by them to learn from. Working on such human organization can provide valuable opportunities to examine the capacity of today's ability for managing tomorrow's likely threats, on the one hand, and unveil the inherent capacities, on the other hand, of these integral contexts for efficiency in energy consumption.

This text summarizes the morphological studies of the project "PolimiparaRocinha", a 2016 Polisocial winner dedicated to the Favela Rocinha which is the biggest single favela in Brazil. PolimiparaRocinha is an interdisciplinary project coordinated by the Department of Architecture, Built Environment and Construction Engineering $(\mathrm{ABC})$ with the Department of Civil and Environmental Engineering (DICA), the Department of Architecture and Urban Studies (DAStU) and the Department of Energy, and several academic and non-academic partners outside the Politecnico di Milano.

Acknowledging the particularities in all of its aspects, there is no doubt that Rocinha is far from ordinary urban settlement. Its placement in the heart of Rio, along with its practical isolation from it, the semi-independent functional distribution within its boundaries, its strong community-oriented social fabric, its informal organic-like morphology that accommodates an astonishing population density, and the bewildering energy consumption statistic associated with it outlines a complex system of multidimensional urban organisms linked to one another in a profound and occasionally paradoxical way producing a wide variation of situations. Studying such a context demands a synthesis-based approach able to address its profound systemic interconnectedness and occult qualities which are the source of both strengths and weaknesses in Rocinha's performance. The interdisciplinary framework of this project should also be structured around an operative holistic methodology used as the departure and reference point for all the defined projects in different realms and areas.

The core methodological tool for the investigation, evaluation, and project definition used in PolimiparaRocinha is Integrated Modification Methodology (IMM). IMM is a procedure encompassing a set of scientific techniques for understanding the systemic structure of urban settlements and proposing modification scenarios 
to enhance their socioeconomic and environmental performances. It was developed by the IMM Design Lab based in the ABC Department of Politecnico di Milano. Based on system thinking, the main purpose of IMM is to introduce modification scenarios in order to morphologically transform the built environment-in different scales-into ecologically better-performing systems.

\section{Integrated Modification Methodology (IMM)}

Integrated modification methodology (IMM) is a procedure encompassing an open set of scientific techniques for morphologically analyzing the built environment in a multiscale manner and evaluating its performance in current states or under specific design scenarios (Vahabzadeh Manesh et al. 2011).

In IMM, the built environment is considered a complex adaptive system (CAS) in which the relationships between the parts are highly complicated in a way that a mere local modification starts a chain reaction and ultimately changes the entire system.

According to system theory, the functioning manner of any system is fundamentally directed mostly by the relationship between the parts and depends less on the quality of its individual components (Wächter 2011).

IMM recognizes the built environment as a complex adaptive system (CAS) comprised of numerous subsets and many variables interacting on various levels, various scales, and a diverse set of subcategories. Rendering the CAS's nature, a mere local action accrued in an individual subset will produce a chain reaction within the network of its parts and trigger a process which consequently leads to the global change of the entire system. In other words, system agents adapt themselves in response to the complex network of reactions arisen from individual changes (Vahabzadeh Manesh and Tadi 2013).

The generic morphological subsystems recognized by IMM are namely: urban built-up, urban void, types of uses, and links (Tadi et al. 2015). In the first phase, they are being investigated individually, and the structural attributes resulting from their fusion is studied. The former distinctively gives an understanding of the component, and the latter quantifies the relational attributes that as previously explained shapes the functioning manner of the context. These steps are called horizontal investigation and vertical investigation, respectively, and unveil the weakest elements and mechanisms mostly responsible for the current performance. The next phase is to assess this analysis and formulate strategies to modify the system based on design ordering principles (DOP). The phase after that is to test the design scenarios through the same procedures that evaluate the existing situation in order to reach the optimum modification plan. The outcome is locally retrofitted in the last phase, and the achievements in performance improvement are reported.

In PolimiparaRocinha, four main categories of challenges are defined: 1. People engagement, 2. Managing the unbalanced density, 3. Proposing waste management strategies, and 4. Proposing water management. For addressing these issues, five 
project themes have been identified: 1. Ecosystem services, 2. Food production, 3. Mobility, 4. Energy, and 5. Waste management (Arcidiacono et al. 2017).

The project activities are initiated with IMM phasing in order to provide a modified morphology flexible enough to achieve maximum performances in all the mentioned project themes.

The following is a summary of the IMM intervention on Rocinha.

\section{Vertical Investigation}

In vertical investigation, the attributes associated with these relationships are to be referred to as key categories. Since the performance of any system is resulted from the relationships between its elements, it is safe to state that vertical investigation is the methodological engine of IMM.

Six key categories have been investigated in Rocinha: Porosity, Proximity, Diversity, Effectiveness, Accessibility, and Interface.

\section{Porosity}

Normally, analyzing porosity in IMM encompasses a certain number of concepts including building coverage, density, volume distribution concentration factor, etc., in a comparable manner. However, because the population density in Rocinha is dramatically high, it is almost impossible to carry out a comparative analysis. Density is the only key player in Rocinha, and no realistic modification scenario could be imagined that could abate this staggering statistic (Tadi and Mohammad Zadeh 2017).

Thus, the porosity investigation in PolimiparaRocinha is a comprehensive study of built-up density which with consideration of integrity in social characteristic in the whole favela could also be directly interpreted as population density.

In this analysis, the buildings have been categorized with regard to their heights. Considering the almost uniform size of the building footprints, the porosity investigation here shows the distribution of density in an acceptably accurate way. Although there are limited numbers of buildings that are up to eight stories, the highest typical buildings are four-story buildings. This typology finds its peak primarily in the vicinity of the western section (because of the metro station) and alongside da Gávea street. The volume distribution in the rest of the favela is following a quasi-random pattern (Fig. 1).

\section{Proximity}

Proximity is the quality by which an urban area's main uses can be reached by means of non-motorized transportation (mainly walking). The main relationship here is the one between functions and volume/voids. Of course, the street network is also a key factor. In order to analyze proximity, according to its definition, it is fundamental to define the key urban functions and to investigate the way in which the functions influence non-motorized mobility (Tadi et al. 2015). 


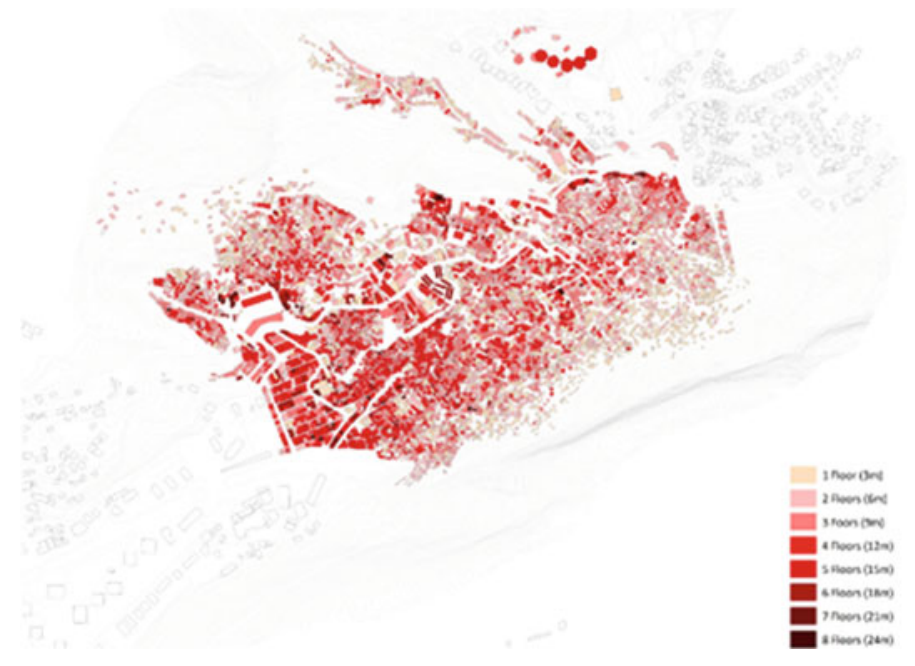

Fig. 1 Porosity in Rocinha

Considering the playful topography of Rocinha and the limited number of functions, the catchment areas have been considered as circles with a radius of 150 meters. However, these circles have been modified by a morphological limit. This means that they have been located with regard to the functions, and the buildings' footprints have been cut away from them in such a way that they are projected onto the voids (this is obvious because people cannot walk through the actual buildings). It is important to notice that the proximity analysis shows the actual/potential walking flow.

The proximity of Rocinha is regulated by the location of the metro and da Gávea street where there are both relatively more functions and enough void spaces. Because of its adequate width and its physical relationship with da Gávea, the walking flow is continued to R. Nova at the center. Occasionally, there are a number of accessible spots in the southern part for walking where the density is medium and the spaces between buildings are enough to support local functions. These scattered patterns indicate a certain level of functional independence due to the significant distance between these areas and the main proximity core.

\section{Diversity}

Similar to the conceptual linkage between open spaces and functions, diversity is about the characteristics of voids influenced by diverging functions. In other words, diversity is the quality of open spaces in giving access to different types of functions (Meurs 2007).

In order to evaluate diversity, IMM as a sustainable-oriented methodology aims at clustering the functions based on travel distances between compatible types of functions. In this regard, if an urban area offers an optimum functional diversity from a social point of view, there is a high chance that an important level of daily needs is met in smaller distances and that helps to avoid unnecessary urban journeys. 


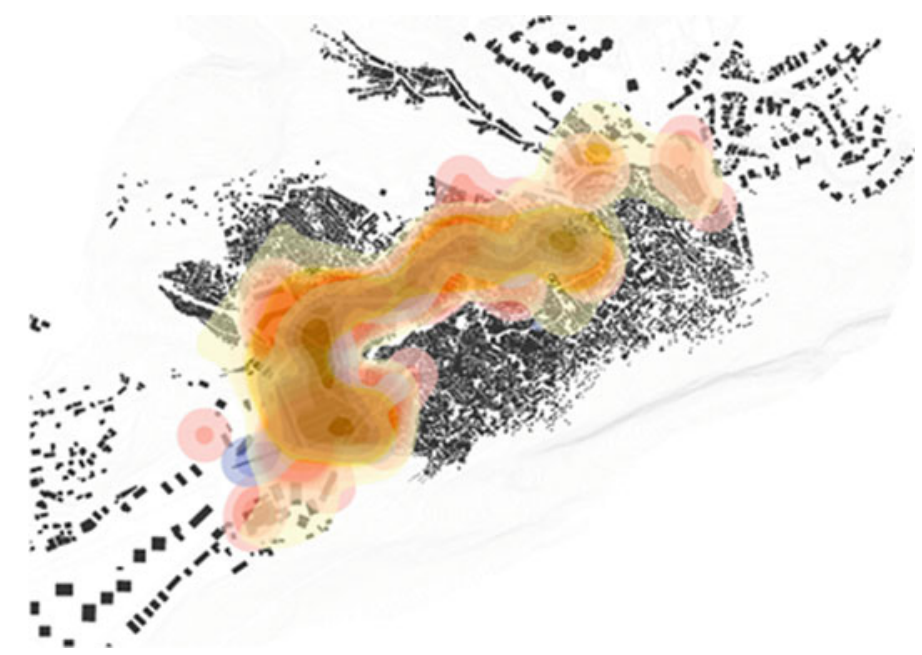

Fig. 2 Diversity in Rocinha

From the social point of view, there are three categories of urban functions: 1 . Necessary activities, 2. Optional activities, and 3. Social activities (Olwig 2016).

Because it is impossible to pinpoint social activities and to involve the time patterns in urban trips, IMM modifies the mentioned categories into: 1. Necessary regular activities, 2 . Necessary occasional activities, and 3. Optional activities.

As it is shown in the diversity analysis of Rocinha, the most diverse parts are again in the areas near the metro station and da Gávea street (Fig. 2).

\section{Accessibility}

Accessibility is the quality of allowing for main functions to be reached via public transportation systems in a certain amount of time. The functional and mobility layers are the boldest urban elements in this key category. To put it simply, the accessibility map illustrates the coverage functions of the public transport stops. The catchment areas are the same as mentioned in effectiveness (Manesh and Tadi 2016).

Because of the Rocinha's simple pattern of functional development around the most accessible areas, it is not shocking that the accessibility analysis is almost identical to the mobility analysis in the horizontal investigation.

\section{Effectiveness}

Mobility and built-ups are the two main building blocks at the heart of effectiveness. As one can observe in its name, this key category is about the effectiveness of the public transportation system. Probably, the most solid way to carry out such an analysis is to study the relationship between population density and public transportation stops. This is to show the density that a stop/station can cover in its walkable catchment areas. According to the literature, this catchment area is a circle (projected to open spaces) with a 400 meters radius for bus and tram stops and 800 meters for metro stations (Handy 2005). 
Below is the effectiveness analysis of Rocinha with regard to the various mobility configurations existing in the favela.

\section{Interface}

IMM interface is evaluated through mean depth calculation and the axial analysis provided by Space Syntax. The axial analysis is a simple iterative computation based on graph theory in which the number of the intersections to reach a certain link is calculated on the basis of all the parts of the street networks. At the end, the links that gain lower depth are the ones which are connected to the system with much higher integrity (Hillier and Iida 2005).

$$
D=\frac{\Sigma d \cdot n}{k-1}
$$

$D$ : mean depth, $d$ : depth, $n$ : number of unit spaces at a specific depth, $k$ : total unit spaces that comprise the system.

The interface analysis vividly illustrates that the street network of Rocinha provides a very low level of connectivity, dictating a low quality of internal movement. It is not surprising that the urban flow is easily interrupted throughout. Interestingly, the parts with low integrity are exactly where criminal groups prefer to arrange their activities. This situation is mainly due to the limitations caused by topography and the irregular pattern of buildings (which itself is an indirect consequence of topography (Fig. 3).

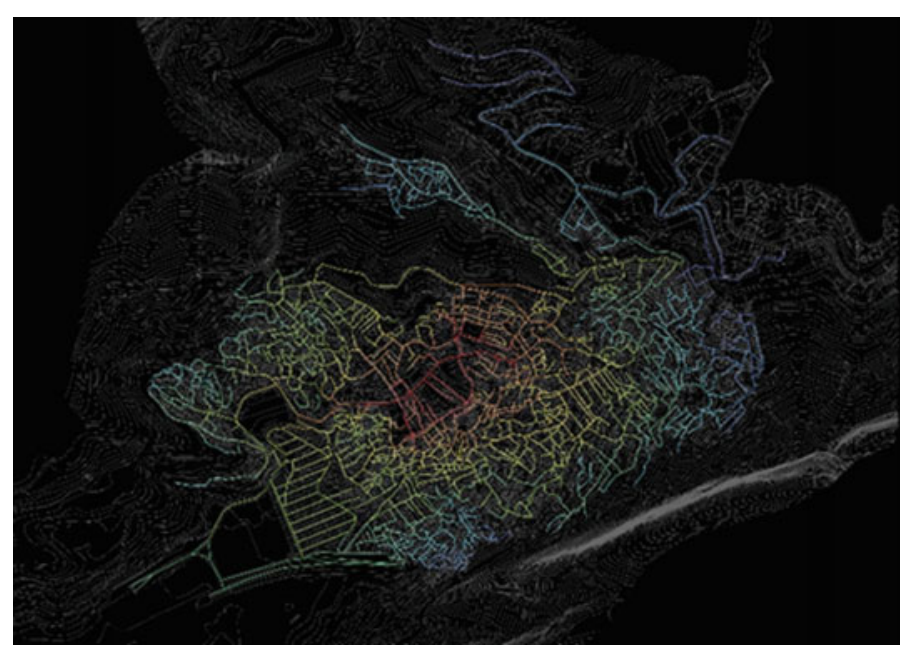

Fig. 3 Interface in Rocinha 


\section{Proposed Morphological Modification}

According to the investigation phase, it is evident that the malfunctioning urban element is the void layer and the most problematic key category is the interface. Rocinha is suffering from not having enough empty spaces to provide sufficient flexibility for urban flow and functional support. On the other hand, the street network is a broken system unable to offer adequate connectivity for smooth movement. However, minor changes can be made to improve the situation and overall functioning of the system.

Accordingly, the initial concept is driven by the idea of providing more open spaces, hence more integrity to the street networks by relocating a small number of low buildings where it is possible and beneficial. A very conceptual change in the interface analysis supports the idea that with a limited local modification, considerable global enhancement can be achieved.

Based on the interface analysis, 21 locations have been identified where the definition of new links resulting from minimum relocation projects could lead to massive global changes in system integration. Accordingly, a total of 108 small buildingswhich in comparison with the whole of Rocinha can be safely considered negligible-were predicted to be relocated to the nearest buildings possible. This decision was entirely supported by the local partners of the project including Sorriso dei miei Bimbi which is an educational institute inside Rocinha and is in close contact with the local community. However, due to specific considerations regarding the social fabric of the different zones, it was suggested that the work be initiated in six specific zones (Fig. 4).

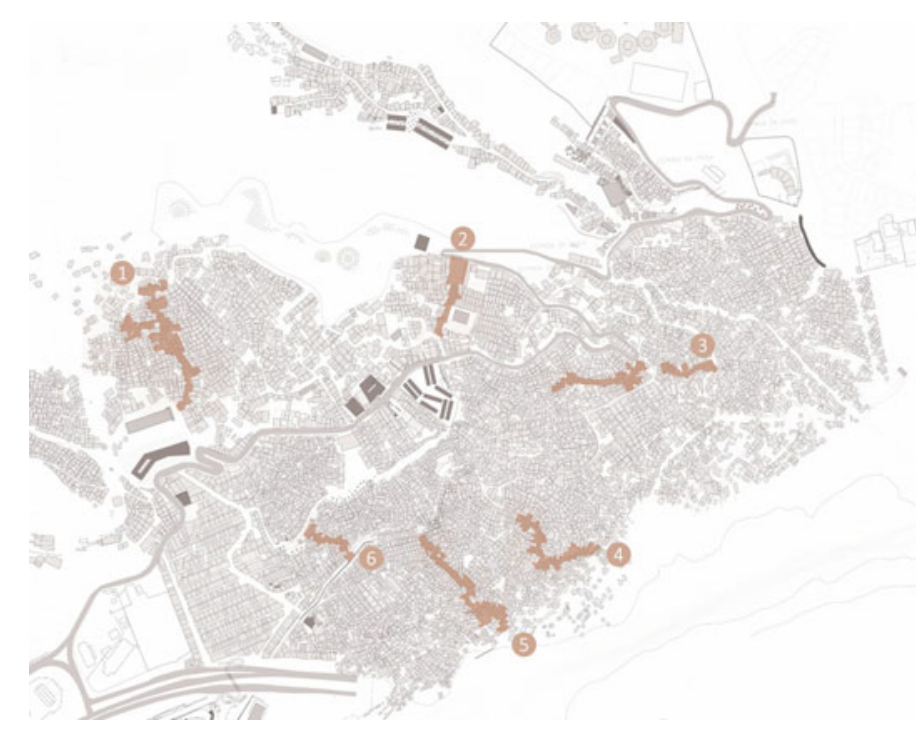

Fig. 4 Intervention zones 
This systemic modification creates an optimum morphological flexibility in view of proceeding with the project themes. The immediate consequence is to have a better mobility flow that not only makes the area safer, but also allowed to define a locally based bicycle network supported by bike-sharing systems which work in compatibility with the existing public transportation system. In some of the new spaces defined by the relocation project, community gardening and aquaponic projects will be established in order to raise awareness on the value of local food production. These projects are integrated with the ecosystem service to ensure the management of runoff and water conservation and the definition of a smart energy grid for harvesting and managing the renewable sources for energy production and management. Local strategies to use organic waste for producing biogas have also been considered, and new waste management plans in compatibility with local programs have been proposed.

It is crucial to address the totality of the structure made by local projects in the selected zones. These six zones are the locations in which local strategies like the aquaponics, photovoltaic panels, community gardening, sewage system, etc., are placed together to create an integrated system of a prototype network throughout Rocinha. This system has been designed in such a way as to ensure two levels of circularities on two different scales. They form a closed system so that their inflow is provided locally. The food production uses local resources, the solar radiation is harvested on top of the local buildings, and the proposed functions are in compatibility with local needs. However, they are linked together all over the favela with the smart grid centrally managed by an urban management system (UMS). The proposed improvements require the development of a specific project which, empowered by the UMS, allows for the circulation of information between citizens, who become the main actors of the whole system, promoting social inclusion and the sustainable regeneration of the favela. The UMS as a system of computer-aided tools will monitor, control, and optimize the information flows coming from the different sectors improving services for citizens such as street lighting, electrical local urban transportation, food delivery, waste management, goods delivery, etc. In this way, the UMS can, for example, reduce traffic in congested areas, encourage the use of more efficient and ecological transport systems, prevent the frequent blackouts as well as establish citizens' virtuous behavior in terms of waste collection, energy savings, etc. (Fig. 5).

On the Rocinha scale, this system creates a balance between inflow and outflow by allocating the local resources to the overall outflow. Moreover, thanks to the prototypes, a new bicycle network has been designed which connects the intervention zones and other places (where the topography allows) physically. This means that local resources provide global energy storage, public lighting, and overall connectivity. This integrity in the prototype network allows us to move from 21 critical locations to be improved to only 6 intervention zones without sacrificing the totality of the favela; nevertheless, the prototype network provides a capacity of integration with more intervention zones applying the same strategies in the future (Fig. 6). 


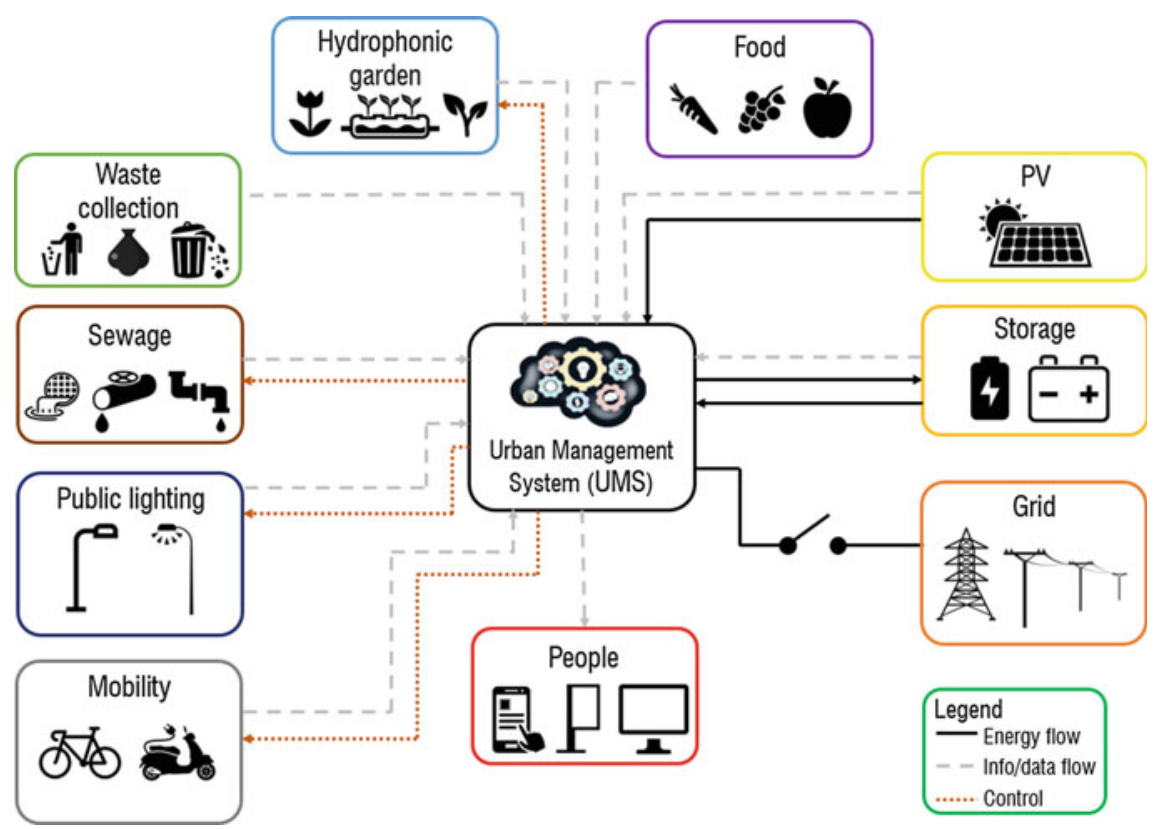

Fig. 5 Urban flow system

\section{Conclusion}

As was explained previously, the relocation project would enable the other subprojects to proceed and create maximum unity between them on different scales. Along with creating new urban spaces and connections in local zones which immediately leads to having a smooth urban flow and a greater level of safety, it raises the ranks of other links throughout Rocinha, which become more integrated. The retrofitting phase clearly showed an advancement in numbers. The modification process in a project of this magnitude and the urban mechanisms quantitatively contributed to revealing the hidden links between the structure and performance, which are naturally measured by different indicators. Such an approach not only helped in pinpointing the key issues in Favela Rocinha and making appropriate decisions, but also provided a new diagnosis system which is measurable, objective, and performance-oriented (Fig. 7).

PolimiparaRocinha is a clear demonstration of how systemic local actions integrated with the whole can produce controlled chain reactions to induce changes on the scale of the entire favela. The proposed sub-projects will not only effectively change the different aspects of urban life in the intervention zone for the better but they will also make tangible improvements to the performance of Rocinha as a whole, even in those areas where the project does not reach. The procedure of interventions proposed by PolimiparaRocinha can easily be replicated for the other parts of the 


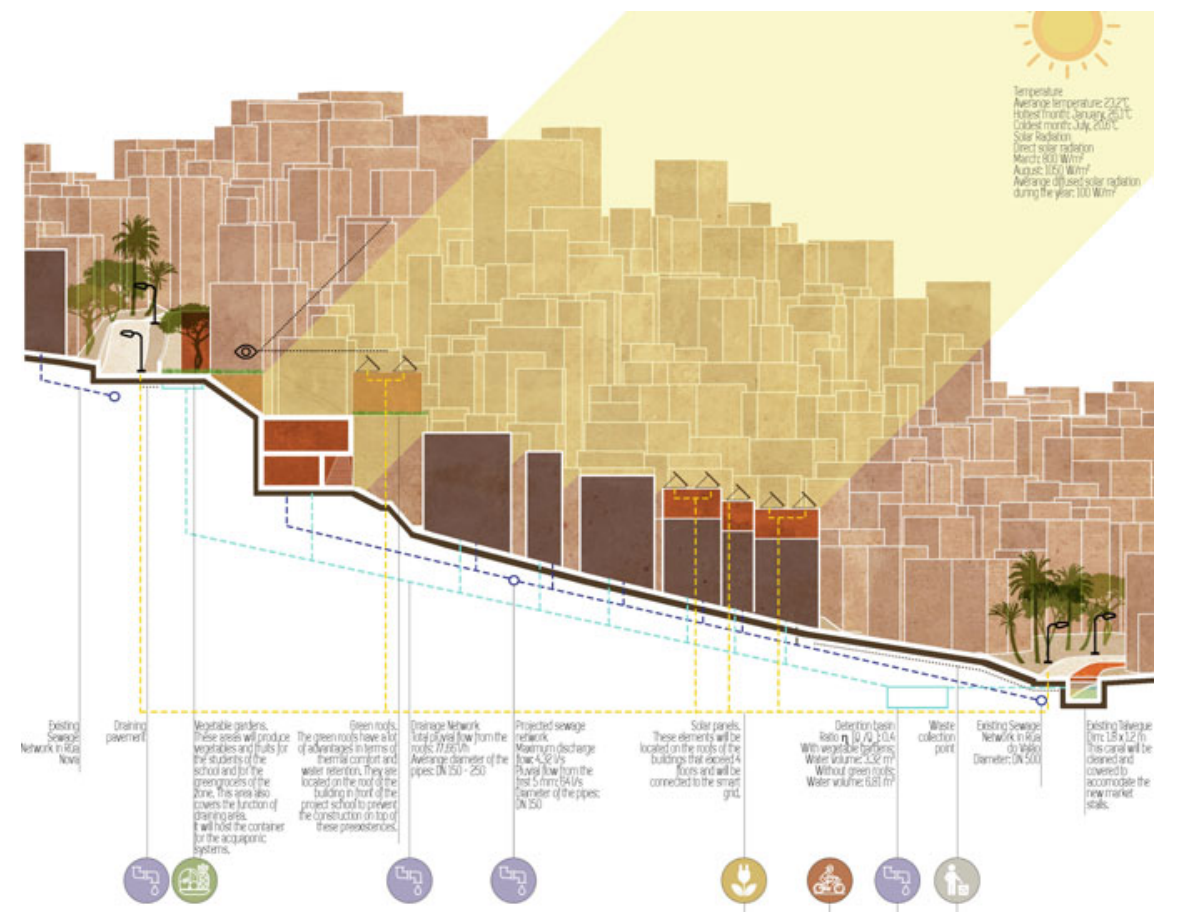

Fig. 6 Local interventions

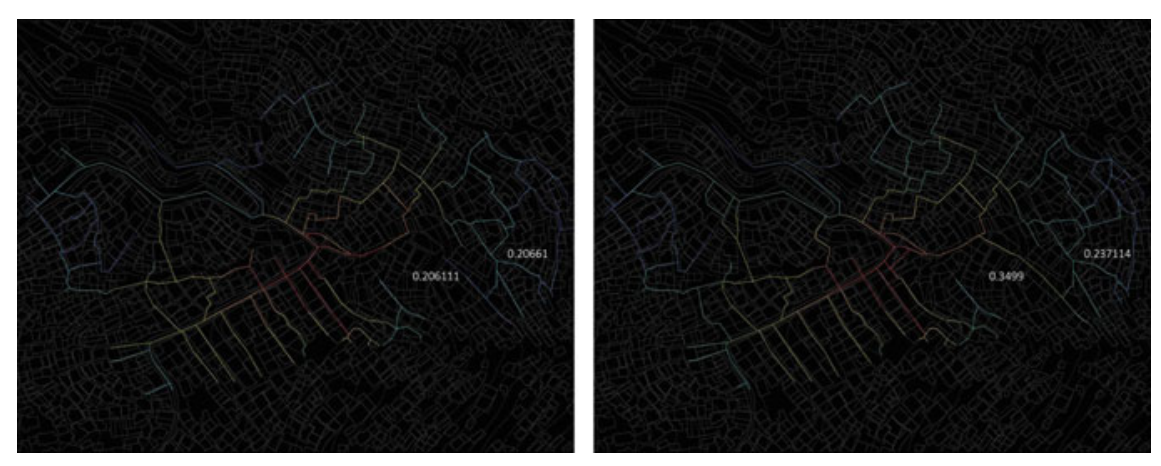

Fig. 7 Local interface of an intervention zone before (left) and after (right) the intervention 
favela and create a high level of integrity which could advance the quality of life and environmental performances therein (Arcidiacono et al. 2017).

The investigation phase highlights the structural blockage in urban flow due to the morphological pattern of Rocinha. Although there were 21 locations in which the morphological modification could create a systemic reaction on a favela-wide scale, an integrated prototype network allowed for the intervention to be applied in only six locations, still activating the same systemic reaction. The UMS designed to control the flow of energy and information is based locally and can include future intervention in Rocinha to integrate this with the whole favela system.

Today, we are facing challenges of unprecedented difficulty such as climate and socioeconomic inequity, which cast a shadow of doubt over our sustainable future. As most of the problems have their roots in cities, sustainability becomes an urban matter. While there should be a collective effort to minimize urban marginalization in future developments, the current problems of these areas should be addressed and effective methods to improve the quality of life within them should be studied. There are indeed favorable traits in the structure of the informal settlements especially in adequate energy consumption which could be learned from.

This project is not the first study proposed to deal with the favela Rocinha or with the informal settlements in general. In most of them, the informal settlements are regarded as a problem to be solved and the efforts were directed to formalize them or to eliminate them from the face of the cities. It is no surprise that they could not relate to the local communities and resulted in producing more conflicts and segregation. In contrast, PolimiparaRocinha is regarding Rocinha as an integral part of the city and a source of opportunity which with a locally based and minimal and systemic set of modifications has the potential to perform better and provide greater opportunities for integration with the rest of the city.

\section{References}

Arcidiacono, A. et al. (2017). Environmental Performance and social inclusion: A project for the Rocinha Favela in Rio de Janeiro. Energy Procedia.

Handy, S. (2005). Smart growth and the transportation-land use connection: What does the research tell us? International Regional Science Review.

Hillier, B., \& Iida, S. (2005). Network and psychological effects in urban movement. In Lecture notes in computer science (including subseries Lecture Notes in Artificial Intelligence and Lecture Notes in Bioinformatics).

Manesh, S. V., Tadi, M. (2013). Sustainable morphological transformation via integrated modification methodology (I.M.M): The case study of surfers Paradise District of Gold Coast City, Australia.

Manesh, S. V., \& Tadi, M. (2016). A sustainable urban morphology for a greener city. The International Journal of Architectonic, Spatial, and Environmental Design.

Meurs, M. (2007). Understanding institutional diversity. Comparative Economic Studies.

Olwig, K. R. (2016). Life between buildings: Using public space. Landscape Journal.

Tadi, M., \& Mohammad Zadeh, M. H. (2017). Urban porosity. A morphological key category for the optimization of the CAS's environmental and energy performances. 
Tadi, M., Vahabzadeh Manesh, S., Mohammad Zadeh, M. H., \& Zaniol, F. (2015). Transforming urban morphology and environmental perfornances via IMM ${ }^{\circledR}$ the case of Porto Maravilha in Rio de Janeiro. GSTF Journal of Engineering Technology.

Vahabzadeh Manesh, S., Tadi, M., \& Zanni, F. (2011). Integrated sustainable urban design: Neighbourhood design proceeded by sustainable urban morphology emergence. WIT Transactions on Ecology and the Environment.

Wächter, P. (2011). Thinking in systems-A primer. Environmental Politics.

Open Access This chapter is licensed under the terms of the Creative Commons Attribution 4.0 International License (http://creativecommons.org/licenses/by/4.0/), which permits use, sharing, adaptation, distribution and reproduction in any medium or format, as long as you give appropriate credit to the original author(s) and the source, provide a link to the Creative Commons license and indicate if changes were made.

The images or other third party material in this chapter are included in the chapter's Creative Commons license, unless indicated otherwise in a credit line to the material. If material is not included in the chapter's Creative Commons license and your intended use is not permitted by statutory regulation or exceeds the permitted use, you will need to obtain permission directly from the copyright holder. 